\title{
Entropy of the diluted antiferromagnetic Ising models on the frustrated lattices using the Wang-Landau method
}

\author{
Yuriy Shevchenko ${ }^{1,2},{ }^{*}$ Konstantin Nefedev $^{1,2},{ }^{\dagger}$ and Yutaka Okabe ${ }^{3 \ddagger}$ \\ ${ }^{1}$ School of Natural Sciences, Far Eastern Federal University, \\ Vladivostok, Russian Federation \\ ${ }^{2}$ Institute of Applied Mathematics, \\ Far Eastern Branch, Russian Academy of Science, \\ Vladivostok, Russian Federation \\ ${ }^{3}$ Department of Physics, Tokyo Metropolitan University, \\ Hachioji, Tokyo 192-0397, Japan
}

(Dated: July 23, 2018)

\begin{abstract}
We use a Monte Carlo simulation to study the diluted antiferromagnetic Ising model on the frustrated lattices including the pyrochlore lattice to show the dilution effects. Using the WangLandau algorithm, which directly calculates the energy density of states, we accurately calculate the entropy of the system. We discuss the nonmonotonic dilution concentration dependence of residual entropy for the antiferromagnetic Ising model on the pyrochlore lattice, and compare it to the generalized Pauling approximation proposed by Ke et al. [Phys. Rev. Lett. 99, 137203 (2007)]. We also investigate other frustrated systems, the antiferromagnetic Ising model on the triangular lattice and the kagome lattice, demonstrating the difference in the dilution effects between the system on the pyrochlore lattice and that on other frustrated lattices.
\end{abstract}

This paper is accepted for publication in Phys. Rev. E

PACS numbers: $\quad 05.50 .+\mathrm{q}, 75.40 . \mathrm{Mg}, 75.50 . \mathrm{Lk}, 64.60 . \mathrm{De}$

\section{INTRODUCTION}

Frustration plays an important role in several scientific fields. The simplest example is the antiferromagnetic (AFM) Ising model on the triangular lattice. One cannot select all three pairs of spins antiparallel in the basic unit of the triangle. It is called frustration. Because of frustration, there is no long-range order, and the frustration leads to a high degeneracy of ground states. The existence of the residual entropy was discussed by Pauling in 1935 for water ice [1].

Recently, spin-ice materials have captured particular attention [2-4], and their exotic physics is a current topic of geometrically frustrated magnets. Prototype materials are the pyrochlores $\mathrm{Dy}_{2} \mathrm{Ti}_{2} \mathrm{O}_{7}$ and $\mathrm{Ho}_{2} \mathrm{Ti}_{2} \mathrm{O}_{7}$. In these materials, the magnetic ions $\left(\mathrm{Dy}^{3+}\right.$ or $\left.\mathrm{Ho}^{3+}\right)$ occupy a pyrochlore lattice of corner-sharing tetrahedra, and the local crystal field environment causes the magnetic moments to orient along the directions connecting the centers of two tetrahedra at low temperatures $[4,5]$. In the low-temperature spin-ice state, the magnetic moments are highly constrained locally and obey the so-called "ice rules": two spins point in and two spins point out of each tetrahedron of the pyrochlore lattice. This two-in-twoout spin configuration resembles the situation of hydrogen atoms in water ice. The measured residual entropy is

\footnotetext{
*Electronic address: shevchenko.ya@dvfu.ru

$\dagger$ Electronic address: nefedev.kv@dvfu.ru

${ }^{\ddagger}$ Electronic address: okabe@phys.se.tmu.ac.jp
}

very close to Pauling's estimate, $(1 / 2) \ln (3 / 2) \mathrm{R}$, where $\mathrm{R}$ is the molar gas constant [3].

The dilution effects on frustration were studied by $\mathrm{Ke}$ et al. [6] for spin-ice materials. The magnetic ions $\mathrm{Dy}^{3+}$ or $\mathrm{Ho}^{3+}$ are replaced by nonmagnetic $\mathrm{Y}^{3+}$ ions. Nonmonotonic zero-point entropy as a function of dilution concentration was observed experimentally, and a generalization of Pauling's theory was discussed [6]. More recently, the detailed experimental studies combined with Monte Carlo simulations were reported [7, 8].

The effect of a magnetic field is another topic of spin-ice materials, and recently, Peretyatko, Nefedev, and Okabe [9] studied the effect of a magnetic field on diluted spinice materials in order to elucidate the interplay of dilution and magnetic field. They observed five plateaus in the magnetization curve of the diluted nearest-neighbor spinice model on the pyrochlore lattice when a magnetic field was applied in the [111] direction. This effect contrasts with the case of a pure (i.e. undiluted) model, which displays two plateaus.

In this paper, motivated by the current interest in the pyrochlore lattice, we study the entropy of the diluted AFM Ising model on the frustrated lattices using the Monte Carlo simulation. If one uses a canonical Monte Carlo simulation such as the Metropolis algorithm, the estimate of the entropy is made by the numerical integration of specific heat. A more straightforward way of computing entropy is to use the Monte Carlo method that directly calculates the energy density of states (DOS) $g(E)$, for example, the Wang-Landau (WL) method [10]. The WL method is an efficient algorithm to calculate $g(E)$ with high accuracy. Several recent progresses have been 


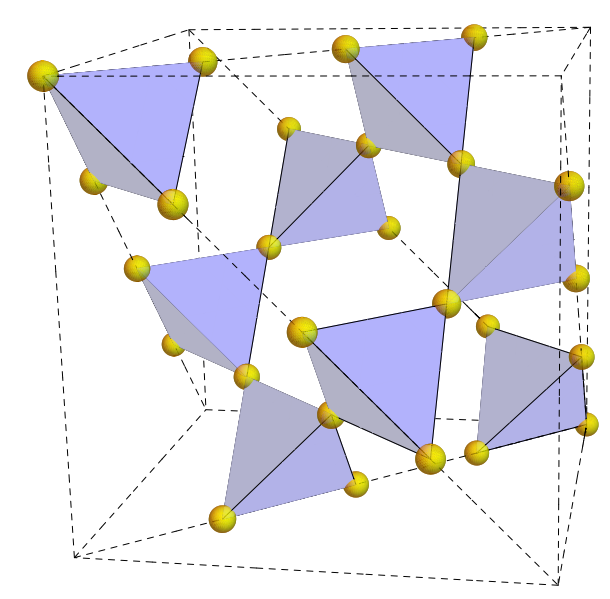

FIG. 1: (Color online) The illustration of the 16-site cubic unit cell of the pyrochlore lattice $(L=1)$.

made in connection with the WL method. To improve the convergence, the $1 / t$ algorithm was proposed [11]. Using the WL method, the difference of the energy DOS was examined to discuss the behavior of the first-order transition [12]. A parallel WL method based on the replica-exchange framework for Monte Carlo simulations was also proposed [13]. Recently, Ferreyra et al. [14] reported the calculation of $g(E)$ for the Ising model on the pyrochlore lattice using the WL method.

As a theoretical model of the spin-ice material, we treat the nearest-neighbor AFM Ising model on the pyrochlore lattice. A more complicated model, such as the dipolar model, may be required to make connections to actual materials. However, Isakov et al. [15] discussed the reason why the low-temperature entropy of the spin-ice compounds is well described by the nearest-neighbor AFM Ising model on the pyrochlore lattice, i.e., by the "ice rules".

The pyrochlore lattice can be regarded as alternating kagome and triangular layers, and the magnetic field in the [111] direction effectively decouples these layers. The triangular and kagome lattices provide the twodimensional frustrated systems. It is interesting to compare the behavior of the dilution effects on the AFM Ising model of the pyrochlore lattice and those of the triangular and kagome lattices.

The purpose of the paper is as follows. First, we obtain precise estimates of entropy for diluted systems using the WL method. Second, we compare the dilution concentration dependence of the residual entropy of the pyrochlore lattice with the generalized Pauling's estimate by Ref. [6]. Third, we uncover the mechanism of the nonmonotonic behavior of residual entropy. Finally, we compare the dilution effects on the residual entropy for several frustrated Ising systems on the pyrochlore, triangular and kagome lattices.

The paper is organized as follows: Section II describes (a)

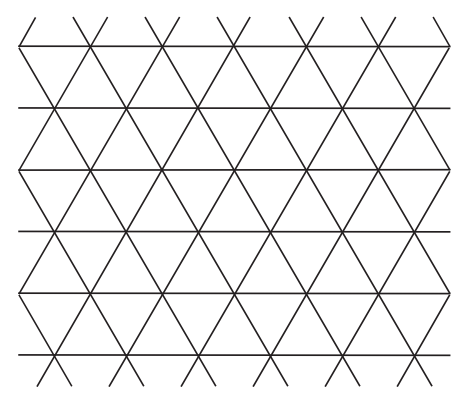

(b)

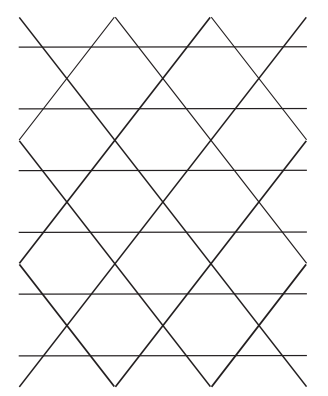

FIG. 2: The illustration of the (a) triangular and the (b) kagome lattice.

the model and the method. The results are presented and discussed in Section III. Section IV is devoted to the summary and discussions.

\section{MODEL AND SIMULATION METHOD}

We investigated the AFM Ising model with nearestneighbor interaction on the pyrochlore lattice. Later, we also treat the Ising model on the triangular and kagome lattices. The pyrochlore lattice is illustrated in Fig. 1, whereas the triangular and kagome lattices are illustrated in Fig. 2.

The Hamiltonian is given by

$$
H=J \sum_{\langle i j\rangle} s_{i} s_{j}, \quad\left(s_{i}= \pm 1\right)
$$

where $\langle i j\rangle$ stands for the nearest-neighbor pairs. Hereafter, the coupling $J$ is set as 1 unless otherwise specified. We are especially interested in the site dilution of spins. Then, the Hamiltonian becomes

$$
H=J \sum_{\langle i j\rangle} c_{i} c_{j} s_{i} s_{j}, \quad\left(c_{i}=1 \text { or } 0\right) .
$$

Here $c_{i}$ is the quenched variable, and the concentration of vacancies is denoted by $x$.

Quenched randomness is investigated basically under two different constraints, a grand-canonical constraint (average density of vacancies fixed) and a canonical constraint (total number of vacancies constant) $[16,17]$. Here, we use the canonical constraint for the site dilution. As for the dilution concentration $x$, we treat $x=$ 0.0 (pure), 0.1, 0.2, 0.3, 0.4, 0.5, 0.6, 0.7, 0.8, and 0.9 .

To get precise numerical information on the entropy of the system, we use the WL method that directly calculates the energy DOS. Let us briefly review the WL algorithm. A random walk in energy space is performed with a probability proportional to the reciprocal of the DOS, $1 / g(E)$, which results in a flat histogram of the energy 


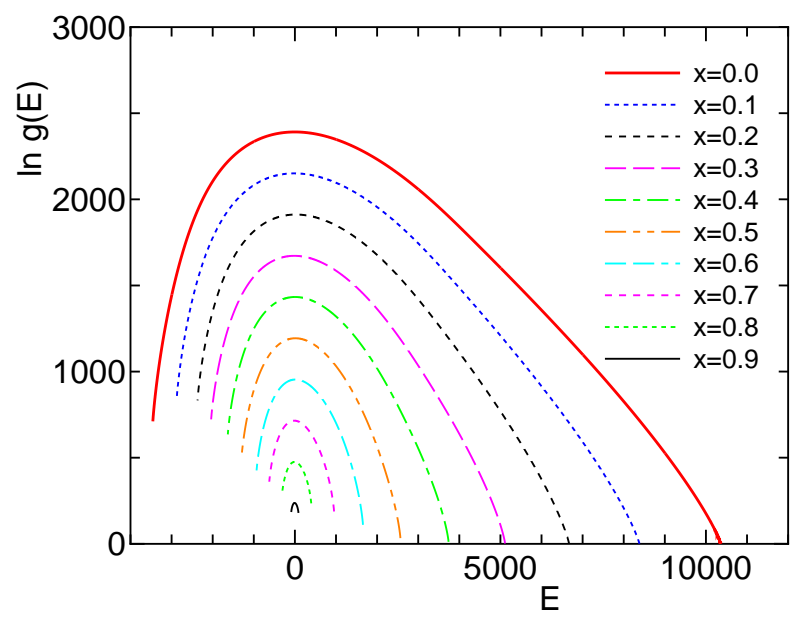

FIG. 3: (Color online) The plot of $\ln g(E)$ as a function of $E$ of the Ising model on the pyrochlore lattice. The system size is $L=6(N=3456)$.

distribution. Actually, we move based on the transition probability from energy level $E_{1}$ to $E_{2}$ :

$$
p\left(E_{1} \rightarrow E_{2}\right)=\min \left[1, \frac{g\left(E_{1}\right)}{g\left(E_{2}\right)}\right]
$$

Since the exact form of $g(E)$ is not known a priori, we determine $g(E)$ iteratively. Introducing the modification factor $f_{i}, g(E)$ is modified by

$$
\ln g(E) \rightarrow \ln g(E)+\ln f_{i}
$$

every time the state is visited. At the same time the energy histogram $h(E)$ is updated as

$$
h(E) \rightarrow h(E)+1 .
$$

The modification factor $f_{i}$ is gradually reduced to unity by checking the "flatness" of the energy histogram. The "flatness" is checked such that the histogram for all possible $E$ is not less than some value of the average histogram, e.g., $80 \%$. Then $f_{i}$ is modified as

$$
\ln f_{i+1}=\frac{1}{2} \ln f_{i},
$$

and the histogram $h(E)$ is reset. As an initial value of $f_{i}$, we choose $f_{0}=e$; as a final value, we choose $\ln f_{i}=2^{-24}$, that is, $f_{24} \simeq 1.00000006$.

\section{RESULTS}

\section{A. pyrochlore lattice}

For the simulation of the Ising model on the pyrochlore lattice, we use the 16-site cubic unit cell of the pyrochlore lattice [18], and the systems with $L \times L \times L$ unit cells

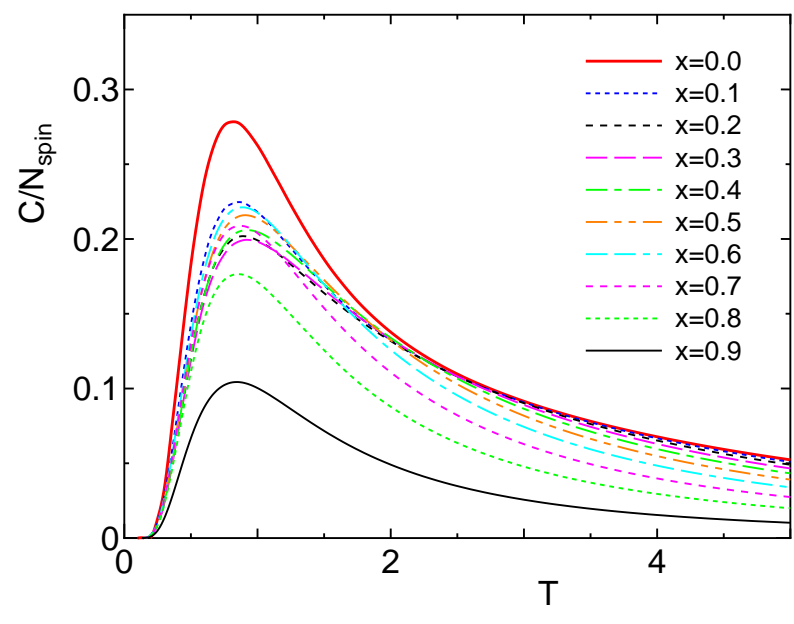

FIG. 4: (Color online) The temperature dependence of the specific heat per spin of the AFM Ising model on the pyrochlore lattice. The system size is $L=6(N=3456)$.

with periodic boundary conditions are treated. We made simulations for the system sizes of $L=3,4,5$, and 6 ; the numbers of sites are $N=432,1024,2000$, and 3456, respectively.

We first show the results of the Ising model on the pyrochlore lattice. In the WL algorithm, one directly calculates the ratio of $g(E)$ for different energies $E_{1}$ and $E_{2}, g\left(E_{1}\right) / g\left(E_{2}\right)$. If we are interested only in the temperature dependence of the total energy or the specific heat, the ratio of $g(E)$ is enough. However, if we discuss the absolute value of entropy, the normalization of $g(E)$ is necessary. In the case of the Ising model, each spin takes one of two states; thus, the normalization condition becomes

$$
\sum_{E} g(E)=2^{N_{\mathrm{spin}}},
$$

where $N_{\text {spin }}$ is the number of spins. In the case of dilution, the number of spins $N_{\text {spin }}$ is different from the number of sites $N$.

We plot $\ln g(E)$, essentially the entropy, as a function of $E$ (in units of $J$ ) of the Ising model on the pyrochlore lattice, shown in Fig. 3. The system size is $L=6(N=$ 3456 ), and this is the plot of one sample for each $x$. The energy takes a value from $-N J$ to $3 N J$ for the pure system $(x=0)$. We note that the energy takes a value of the multiple of $4 J$ for the pure system. For diluted systems, the energy takes a value of the multiple of $J$, but the energy difference of a single spin flip is a multiple of $2 J$. That is, the total energy is either the even number of $J$ or the odd number of $J$.

The thermal average of a physical quantity $A$ at the inverse temperature, $\beta=1 / T$, is calculated from the knowledge of the energy DOS as

$$
\langle A\rangle_{\beta}=\frac{\sum_{E} e^{-\beta E} A(E) g(E)}{\sum_{E} e^{-\beta E} g(E)} .
$$




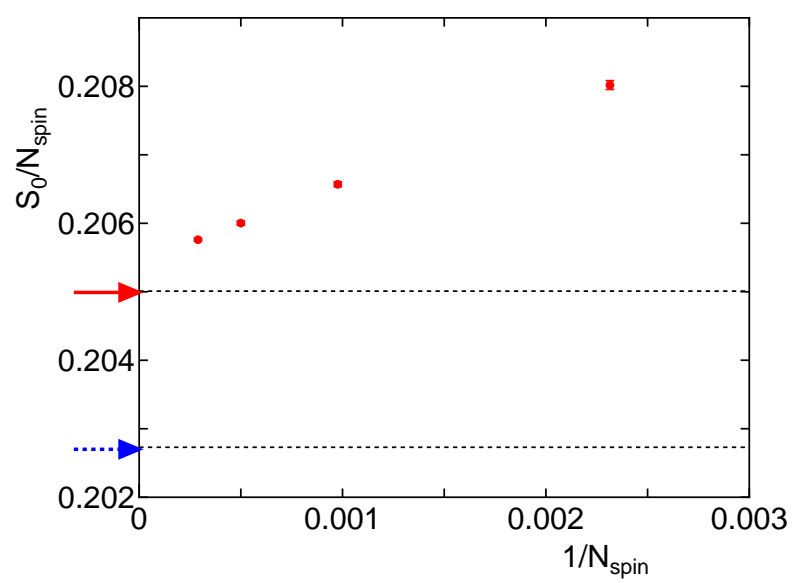

FIG. 5: (Color online) The plot of the residual entropy per spin of the pure AFM Ising model on the pyrochlore lattice as a function of $1 / N\left(N=N_{\text {spin }}\right)$. The system size is $L=$ $3(N=432), L=4(N=1024), L=5(N=2000)$ and $L=$ $6(N=3456)$. The precise estimate by Nagle [19] (0.20501) is shown by the solid red arrow, whereas the approximation by Pauling [1] (0.20273) is shown by the dotted blue arrow.

Then, the specific heat is calculated through the relation

$$
C=\frac{d\langle E\rangle_{\beta}}{d T}=\beta^{2}\left(\left\langle E^{2}\right\rangle_{\beta}-\langle E\rangle_{\beta}^{2}\right) .
$$

The temperature dependence of the specific heat per spin of the AFM Ising model on the pyrochlore lattice $(x=0.0,0.1, \cdots, 0.9)$ is plotted in Fig. 4. The temperature $T$ is measured in units of $J$. The system size is $L=6(N=3456)$. The average was taken over 40 random samples. The statistical errors are smaller than the thickness of the curves. We only show the data of $L=6$. For large enough system sizes of $L=4,5$, and 6 , the size dependence is small, as in the scale of the plot in Fig. 4, because there is no phase transition associated with the long-range order. We should note that the peak of the specific heat becomes lower when $x$ is raised from 0 .

Before discussing the thermal average of the entropy, we checked the accuracy of the calculation of the entropy in detail. The residual entropy can be calculated from the raw data of $\ln g(E)$, which is shown in Fig. 1. We plot the residual entropy for the pure system $(x=0)$ as a function of $1 / N \quad\left(N=N_{\text {spin }}\right)$ in Fig. 5. The precise estimate of the residual entropy of a pure system $(x=0)$ using the series method by Nagle [19] is 0.20501 , which is close to Pauling's estimate $(1 / 2) \ln (3 / 2)=0.20273[1]$. We also observed that the residual entropy approaches the precise estimate by Nagle [19]. This is the same plot as Fig. 4 of Ferreyra et al. [14].

We calculate the thermal average of the entropy by

$$
S=\frac{\langle E\rangle_{\beta}}{T}+\ln \left(\sum_{E} e^{-\beta E} g(E)\right) .
$$

The temperature dependence of entropy per spin of the AFM Ising model on the pyrochlore lattice $(x=$

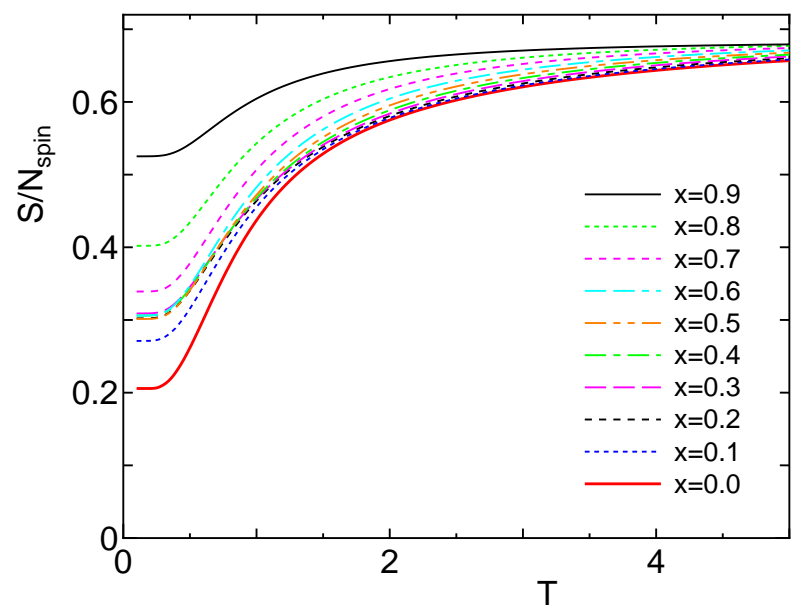

FIG. 6: (Color online) The temperature dependence of the entropy per spin of the AFM Ising model on the pyrochlore lattice. The system size is $L=6(N=3456)$.

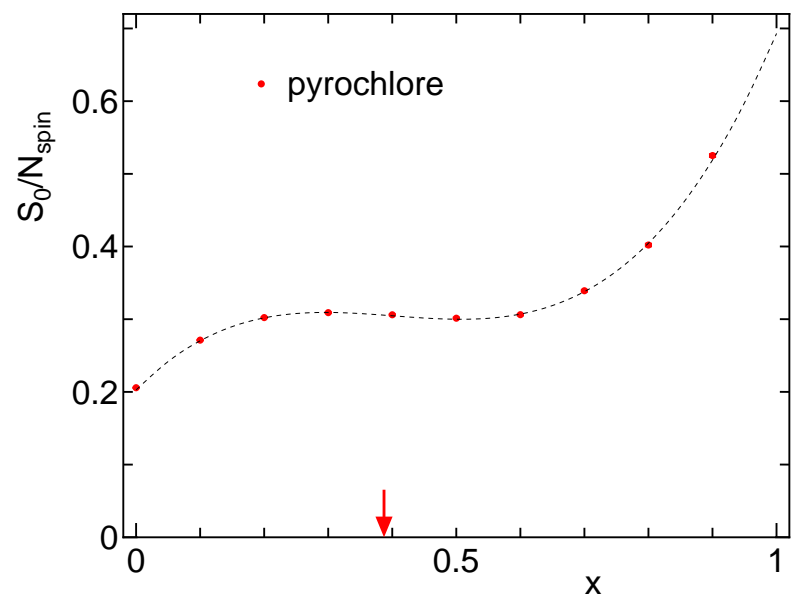

FIG. 7: (Color online) The dilution concentration dependence of the residual entropy per spin of the AFM Ising model on the pyrochlore lattice. The system size is $L=6(N=3456)$. The generalized Pauling approximation [6] is also plotted by a dotted curve. The site-percolation threshold of the pyrochlore lattice is shown by the red arrow.

$0.0,0.1, \cdots, 0.9)$ is plotted in Fig. 6 . The average was taken over 40 samples. The system size is $L=6(N=$ $3456)$, and the size dependence is small as mentioned before. We note that, as $T \rightarrow 0$, the residual entropy becomes larger when $x$ is raised from 0 . In the hightemperature limit where $T \rightarrow \infty$, the entropy per spin approaches $\ln 2=0.693$.

In Fig. 7, we plot the dilution concentration $(x)$ dependence of the residual entropy per spin. The system size is $L=6(N=3456)$. The average was taken over 40 random samples. The statistical errors are smaller than the size of the marks. The size dependence is very small, 
TABLE I: The dilution concentration $(x)$ dependence of residual entropy per spin for the pyrochlore lattice. The system sizes are $L=4,5$, and 6 . The numbers in parentheses are the one-sigma uncertainty for the last digits.

\begin{tabular}{lllll}
\hline \hline$x$ & $\begin{array}{l}L=4 \\
(N=1024)\end{array}$ & $\begin{array}{l}L=5 \\
(N=2000)\end{array}$ & $\begin{array}{l}L=6 \\
(N=3456)\end{array}$ & $\begin{array}{l}\text { Peneralized } \\
\text { Pauling [6] }\end{array}$ \\
\hline 0.0 & $0.2066(0)$ & $0.2060(0)$ & $0.2058(0)$ & 0.2027 \\
0.1 & $0.2736(6)$ & $0.2714(5)$ & $0.2712(4)$ & 0.2703 \\
0.2 & $0.3039(10)$ & $0.3018(10)$ & $0.3023(6)$ & 0.3019 \\
0.3 & $0.3096(15)$ & $0.3101(9)$ & $0.3091(7)$ & 0.3094 \\
0.4 & $0.3043(15)$ & $0.3053(13)$ & $0.3061(8)$ & 0.3047 \\
0.5 & $0.2990(19)$ & $0.3022(12)$ & $0.3014(10)$ & 0.3000 \\
0.6 & $0.3084(24)$ & $0.3065(14)$ & $0.3063(10)$ & 0.3071 \\
0.7 & $0.3373(25)$ & $0.3370(21)$ & $0.3392(13)$ & 0.3380 \\
0.8 & $0.4048(30)$ & $0.4025(25)$ & $0.4021(19)$ & 0.4046 \\
0.9 & $0.5242(40)$ & $0.5231(29)$ & $0.5252(20)$ & 0.5190 \\
\hline
\end{tabular}

and the size difference is not appreciable with this scale of plot. We observed the nonmonotonic concentration dependence of the residual entropy, which was experimentally reported [6, 7]. We also plot the generalized Pauling approximation [6]:

$$
\begin{aligned}
\frac{S_{0}(x)}{N_{\text {spin }}} & =\ln 2+3 \ln (1 / 2)(1-x) x^{2}+2 \ln (3 / 4)(1-x)^{2} x \\
& +(1 / 2) \ln (3 / 8)(1-x)^{3}
\end{aligned}
$$

displayed by a dotted curve. In order to show the numerical data explicitly, we tabulate the measured values of the residual entropy for $L=4,5$, and 6 in Table I. The numbers in parentheses are the one-sigma uncertainty for the last digits, which was estimated by averaging over 40 samples. The statistical errors for $x \neq 0$ come from the average over random samples, whereas they are only for random numbers of simulation for $x=0$, which are very small.

The obtained values agree with the generalized Pauling approximation [6] to three digits for most of the range of $x$, indicating that it is essential for the argument based on the fraction of the configurations of a tetrahedron which satisfy the ground state condition. For the weak dilution region, the residual entropy becomes larger when $x$ is raised from 0 . When one spin is missing from a tetrahedron, there are still 6 lowest-energy configurations out of the 8 possible configurations. This proportion is larger than the undeleted case that there are 6 lowest-energy configurations out of the 16 possible configurations in the tetrahedron. For the strong dilution limit $(x \rightarrow 1)$, the residual entropy per spin approaches $\ln 2(=0.693)$, because all the spins become free. We conclude that the nonmonotonic dilution concentration dependence is primarily accounted for by the nearest-neighbor frustration interaction of the basic tetrahedron unit.

Analyzing the dilution concentration dependence of the specific-heat peak shown in Fig. 4, we also observed that the specific-heat peak shows the nonmonotic dependence. The peak decreases as a function of $x$ for small $x$, increases slightly, and then decreases again. It is anticorrelated with the behavior of the residual entropy due to the increase of the entropy with the temperature, as calculated by

$$
\Delta S=\int\left(\frac{C}{T}\right) d T .
$$

This behavior of the specific-heat peak was previously reported by Ref. [7].

\section{B. triangular and kagome lattices}

To elucidate the nonmonotonic dilution effects on the frustration of the pyrochlore lattice, a comparison with other frustrated systems will be interesting. For this purpose, we studied the AFM Ising model on the twodimensional triangular lattice. The exact solution of this model was given by Wannier [20], who established that this system has no long-range order due to frustration at all the temperatures. The residual entropy of the AFM Ising model on the triangular lattice was calculated to be $0.323066[20]$ :

$$
\frac{S_{0}}{N}=\frac{2}{\pi} \int_{0}^{\pi / 3} \ln (2 \cos \omega) d \omega=0.323066
$$

The dilution effects of the AFM Ising model on the triangular lattice was studied by Yao [21] using the WL method. However, a systematic study was not made for the dilution concentration dependence over the full range.

We carried out the WL study of the AFM Ising model on the triangular lattice. We use the $L \times L$ system with the periodic conditions, and the system sizes are $L=24$ $(N=576), L=32(N=1024)$, and $L=48(N=2304)$. The simulation conditions were the same as those used on the pyrochlore lattice. We again averaged over 40 random samples for each size $L$ and for each dilution concentration $x$.

We plot $\ln g(E)$ as a function of $E$ of the Ising model on the triangular lattice, shown in Fig. 8. The system size is $L=48(N=2304)$, and this is the plot for one sample. The energy DOS $g(E)$ is normalized as $\sum_{E} g(E)=2^{N_{\text {spin }}}$, where $N_{\text {spin }}$ is the number of spins. The energy takes a value from $-N J$ to $3 N J$ for the pure system.

Using the data of the energy DOS, we plot the temperature dependence of the specific heat per spin of the AFM Ising model on the triangular lattice $(x=$ $0.0,0.1, \cdots, 0.9)$, seen in Fig. 9. We plot the data of the system size $L=48(N=2304)$. The size dependence is very small for large enough sizes, $L=24(N=576)$, $L=32(N=1024)$, and $L=48(N=2304)$. The peak of the specific heat becomes higher when $x$ is raised from 0 , which was also shown by Yao [21]. 


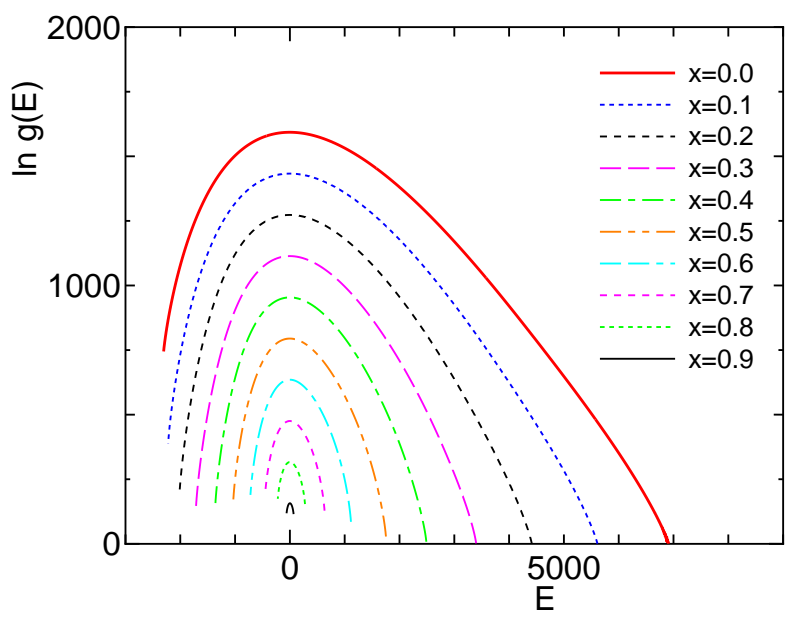

FIG. 8: (Color online) The plot of $\ln g(E)$ as a function of $E$ of the Ising model on the triangular lattice. The system size is $L=48(N=2304)$.

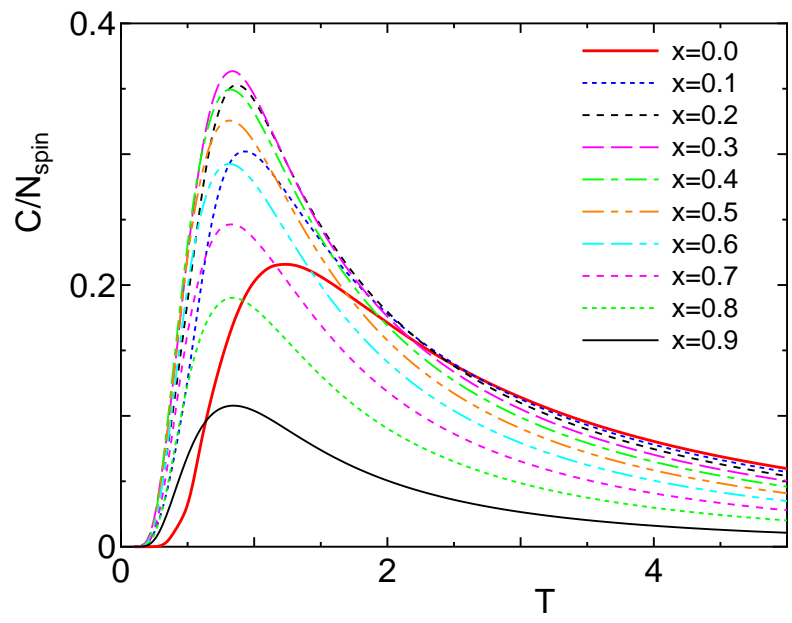

FIG. 9: (Color online) The temperature dependence of the specific heat per spin of the AFM Ising model on the triangular lattice. The system size is $L=48(N=2304)$.

The size dependence of the residual entropy of the pure AFM Ising model on the triangular lattice is given in Fig. 10. Wannier [20] exactly solved the AFM Ising model on the triangular lattice, and the residual entropy of a pure system $(x=0)$ is 0.323066 . We plot $S_{0} / N_{\text {spin }}$ as a function of $1 / N_{\text {spin }}$. Since there is an oscillatory behavior, we also plot the data for $L=64$, observing that the residual entropy per spin approaches Wannier's exact value in the limit as $N_{\text {spin }} \rightarrow \infty$.

The temperature dependence of the entropy of the AFM Ising model on the triangular lattice $(x=$ $0.0,0.1, \cdots, 0.9)$ is plotted in Fig. 11 . We plot the data of the system size $L=48(N=2304)$. Here, the size dependence is very small and the residual entropy becomes

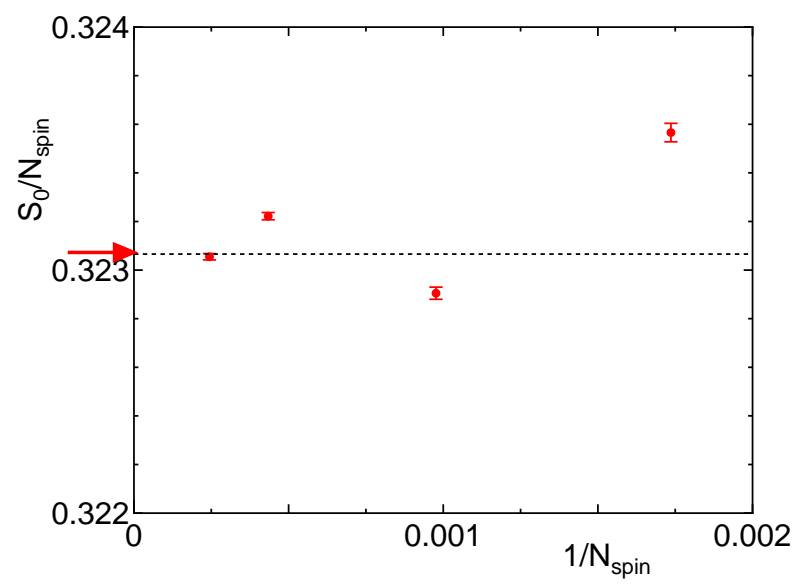

FIG. 10: (Color online) The plot of the residual entropy per spin of the pure Ising model on the triangular lattice as a function of $1 / N\left(N=N_{\text {spin }}\right)$. The system size is $L=24(N=$ $576), L=32(N=1024), L=48(N=2304)$, and $L=$ $64(N=4092)$. The exact value by Wannier [20] (0.323066) is shown by the red arrow.

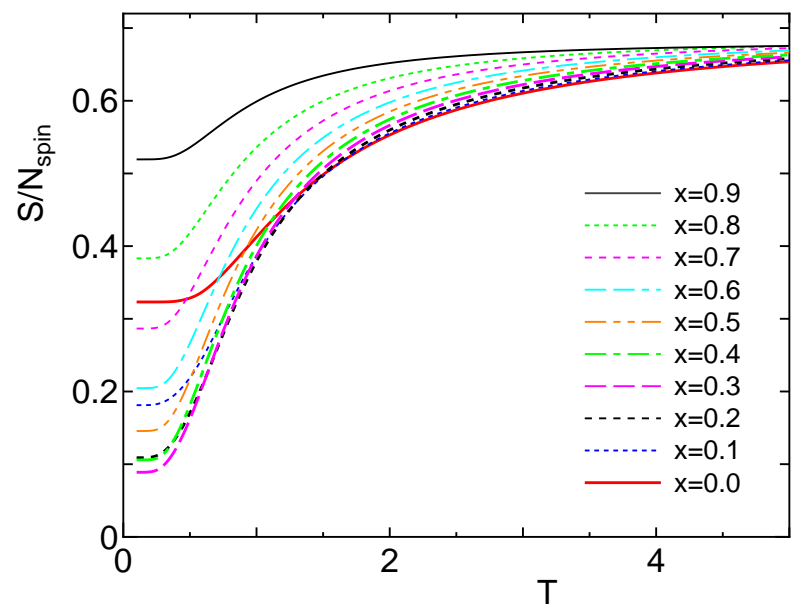

FIG. 11: (Color online) The temperature dependence of the entropy per spin of the AFM Ising model on the triangular lattice. The system size is $L=48(N=2304)$.

smaller when $x$ is raised from 0 , which was also shown by Yao [21].

The dilution concentration $(x)$ dependence of the residual entropy per spin of the AFM Ising model on the triangular lattice is given in Fig. 12. The system size is $L=48(N=2304)$. The residual entropy becomes smaller when $x$ is raised from 0 , which is contrary to the results of the pyrochlore lattice. In the triangular lattice case, the frustration in the basic unit of the triangle is resolved if one spin is deleted from 3 sites. Since the frustration is partially resolved, the degeneracy of the ground states becomes smaller. In the case of the tetrahedron, on the contrary, there still remains a frustration even if 


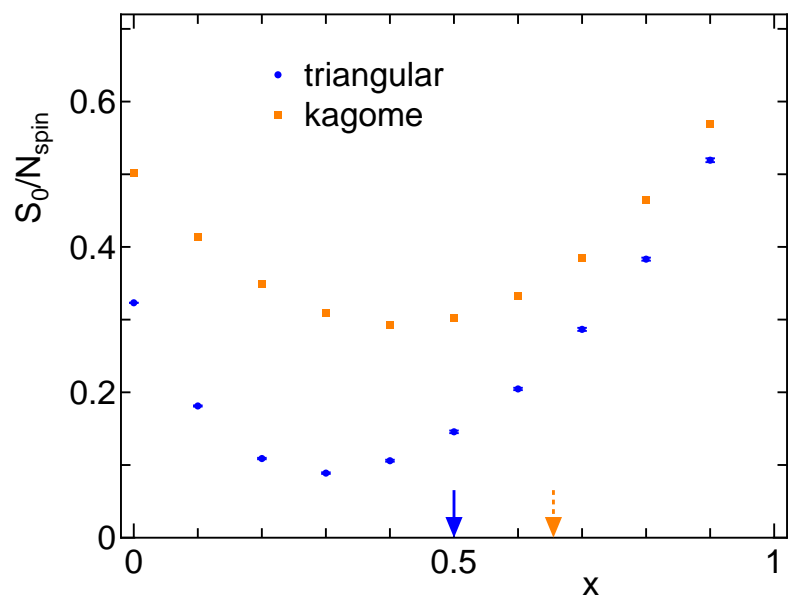

FIG. 12: (Color online) The dilution concentration dependence of the residual entropy per spin of the AFM Ising model on the triangular lattice and the kagome lattice. The system size is $L=48(N=2304$ for the triangular lattice and $N=3456$ for the kagome lattice). The site-percolation thresholds of the triangular and kagome lattices are shown by the solid blue and dotted orange arrows, respectively.

one spin is deleted from 4 sites. For the strong dilution limit $(x \rightarrow 1)$, the residual entropy per spin approaches $\ln 2(=0.693)$ because all the spins become free.

To study another frustrated system, we also considered the AFM Ising model on the kagome lattice. The pyrochlore lattice can be viewed as an alternating stacking of kagome layers and sparse triangular layers. The macroscopic degeneracy of the kagome layers was studied when the magnetic field is applied along the [111] direction [22].

The AFM Ising model on the kagome lattice was exactly solved by Kano and Naya [23]. It was shown that this system has no long-range order due to frustration for all the temperatures. The residual entropy of the AFM Ising model on the kagome lattice was calculated to be $0.50183[23]$ :

$$
\begin{aligned}
\frac{S_{0}}{N}= & \frac{1}{24 \pi^{2}} \int_{0}^{2 \pi} \int_{0}^{2 \pi} \ln \left[21-4\left(\cos \omega_{1}+\cos \omega_{2}+\right.\right. \\
& \left.\left.\cos \left(\omega_{1}+\omega_{2}\right)\right)\right] d \omega_{1} d \omega_{2}=0.50183 .
\end{aligned}
$$

In the WL study of the AFM Ising model on the kagome lattice, we treated the kagome lattice of $L \times$ $(3 / 2) L$, and the system sizes are $L=24(N=864)$, $L=32(N=1536)$, and $L=48(N=3456)$. We averaged over 40 random samples for each size $L$ and for each dilute concentration $x$.

We plot $\ln g(E)$ as a function of $E$ of the AFM Ising model on the kagome lattice, shown in Fig. 13. The system size is $L=48(N=3456)$, and this is the plot for one sample. The energy DOS $g(E)$ is normalized as $\sum_{E} g(E)=2^{N_{\text {spin }}}$, where $N_{\text {spin }}$ is the number of spins. The energy takes a value from $-(2 / 3) N J$ to $2 N J$ for the

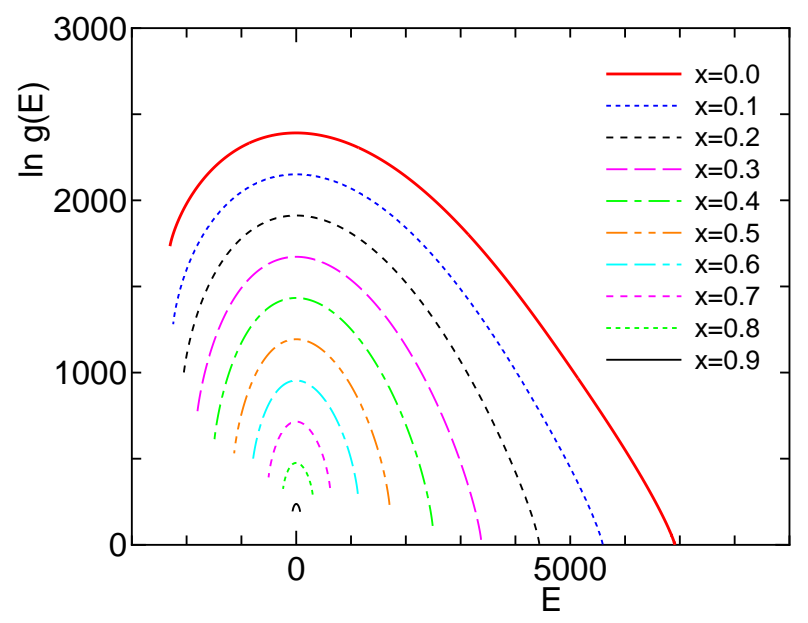

FIG. 13: (Color online) The plot of $\ln g(E)$ as a function of $E$ of the Ising model on the kagome lattice. The system size is $L=48(N=3456)$.

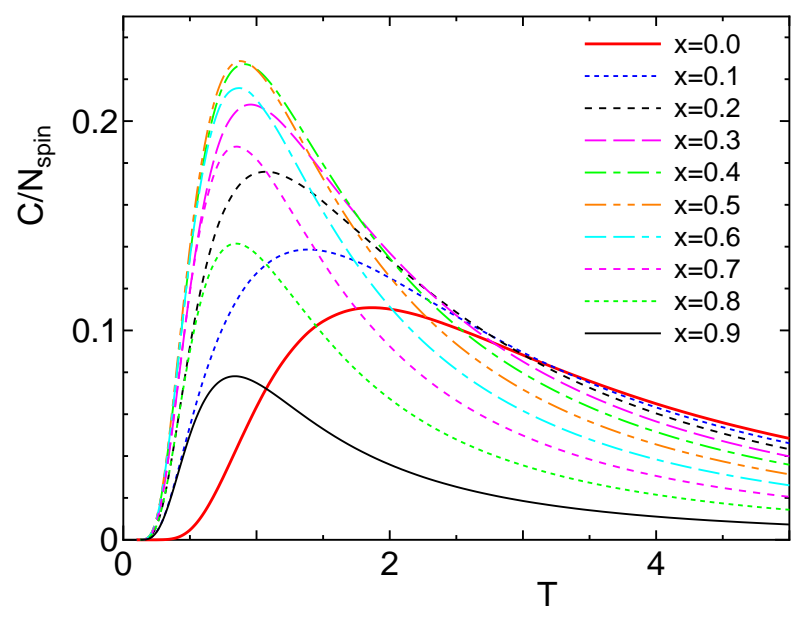

FIG. 14: (Color online) The temperature dependence of the specific heat per spin of the AFM Ising model on the kagome lattice. The system size is $L=48(N=3456)$.

pure system.

The temperature dependence of the specific heat of the AFM Ising model on the kagome lattice $(x=$ $0.0,0.1, \cdots, 0.9)$ is plotted in Fig. 14. Averaging was performed over 40 samples. We plot the data for the system size $L=48(N=3456)$. The size dependence is very small, and the peak of the specific heat becomes higher when $x$ is raised, same as in the triangular lattice.

To confirm the accuracy of our calculation, we plot the size dependence of the residual entropy as a function of $1 / N_{\text {spin }}$ for the pure AFM Ising model on the kagome lattice in Fig. 15. Our calculated result for the residual entropy of the AFM Ising model on the kagome lattice approaches the exactly calculated value given by Kano and Naya as 0.50183 [23]. 


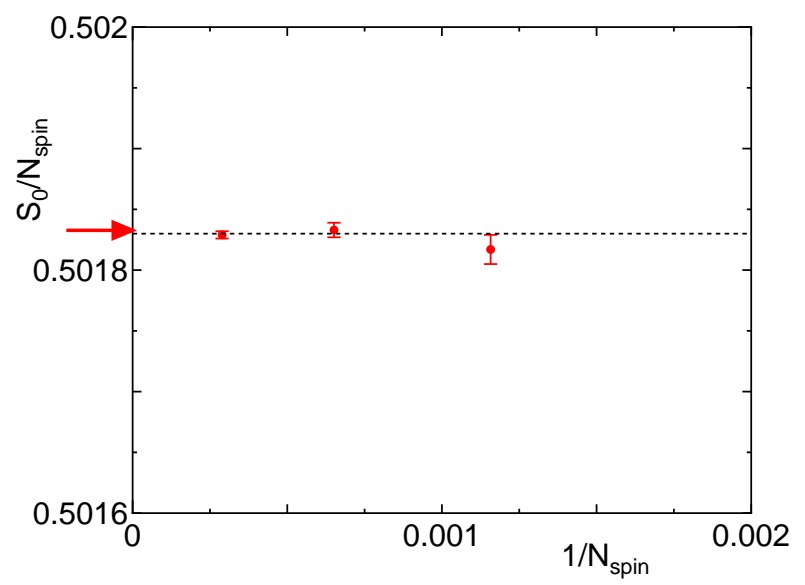

FIG. 15: (Color online) The plot of the residual entropy per spin of the pure Ising model on the kagome lattice as a function of $1 / N \quad\left(N=N_{\text {spin }}\right)$. The system size is $L=24(N=$ $864), L=32(N=1536)$, and $L=48(N=3456)$. The exact value by Kano and Naya [23] (0.50183) is shown by the red arrow.

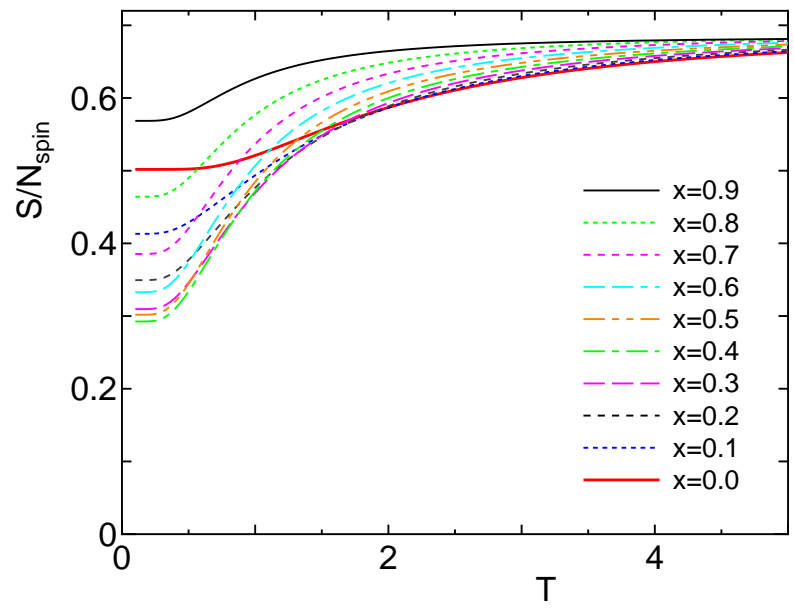

FIG. 16: (Color online) The temperature dependence of the entropy per spin of the AFM Ising model on the kagome lattice. The system size is $L=48(N=3456)$.

The temperature dependence of the entropy of the AFM Ising model on the kagome lattice $(x=$ $0.0,0.1, \cdots, 0.9)$ is shown in Fig. 16. We plot the data for the system size $L=48(N=3456)$. The size dependence is very small, and the residual entropy becomes smaller when $x$ is raised from 0 , exhibiting the same behavior as in the triangular lattice.

We plot the dilution concentration $(x)$ dependence of the residual entropy per spin of the AFM Ising model on the kagome lattice, also shown in Fig. 12. The system size is $L=48(N=3456)$. The residual entropy becomes smaller when $x$ is raised from 0 , the same as that of the triangular lattice. The frustration comes from a triangle in the case of the kagome lattice. Thus, the frustration is resolved when one spin is deleted from this triangle.

\section{SUMMARY AND DISCUSSIONS}

We studied the diluted antiferromagnetic Ising model on the pyrochlore lattice. Using the Wang-Landau algorithm, which directly calculates the energy density of states, we calculated the entropy of the system with a high level of accuracy. We discussed the nonmonotonic dilution concentration dependence of the residual entropy, and obtained a very good comparison with the generalized Pauling approximation proposed by Ke et al. [6].

We also investigated other frustrated systems, the antiferromagnetic Ising model on the triangular lattice and the kagome lattice. We showed the difference in the dilution effects between the system on the pyrochlore lattice and that on other frustrated lattices. The dilution concentration dependence of the residual entropy was compared in Fig. 7 and Fig. 12. For the pyrochlore lattice, the residual entropy increases with $x$ for a weak dilution. When one spin is deleted from the tetrahedron, there still remains the frustration from the other three spins. However, for the triangular and kagome lattices, the residual entropy decreases with $x$. When one spin is deleted from the triangle, the frustration is resolved. The decrease of the residual entropy for the AFM Ising model on the triangular lattice up to $x \leq 0.15$ was shown in Ref. [21], but to the best of our knowledge, no reports have been made for the diluted AFM Ising model on the kagome lattice.

In Fig. 7 and Fig. 12, we also give the values of the site-percolation thresholds. The site-percolation thresholds of the pyrochlore lattice, the triangular lattice, and the kagome lattice are $0.39 \cdots[24], 0.5(=1 / 2)$, and $0.6527 \cdots(=1-2 \sin (\pi / 18))$ [25], respectively. We may say that above the percolation threshold, the residual entropy increases and approaches the free-spin value, $\ln 2=0.691$. In the argument of the generalized Pauling approximation [6], only the local structure within the tetrahedron was considered, but it yields a good comparison with the numerical data. Thus, the percolation threshold is seen not to be directly related to the dilution concentration dependence of the residual entropy.

Based on the high-accurate calculation of the entropy, together with the comparative study of other frustrated systems, we conclude that the nonmonotonic dilution concentration dependence of the residual entropy of the pyrochlore lattice is primarily accounted for by the nearest-neighbor frustration interaction of basic tetrahedron unit.

The long-range dipole interaction may play a role in the detailed comparisons with experimental results. Lin et al. [7] reported the comparative studies of the results of experiments and (canonical) Monte Carlo simulations, which showed a difference between Dy and Ho systems. There was still a discrepancy between the experimen- 
tal and Monte Carlo results. An accurate Wang-Landau study on the pyrochlore system that includes the next nearest-neighbor interaction is still needed, and it will be left to a future work.

Quite recently, the topological spin glass behavior has been discussed for diluted spin ice [26]. The relevance of our results to their study will be interesting, but for now is an open question.

\section{Acknowledgment}

We thank Vitalii Kapitan and Hiromi Otsuka for valuable discussions. The computer cluster of Far Eastern
Federal University was used for computation. This work was supported by a Grant-in-Aid for Scientific Research from the Japan Society for the Promotion of Science, Grant Nos. JP25400406, JP16K05480. This work was also financially supported by a grant of the President of the Russian Federation for young scientists and graduate students, in accordance the Program of development priority direction "Strategic information technology, including the creation of supercomputers and software development".
[1] L. Pauling, J. Am. Chem. Soc. 57, 2680 (1935).

[2] M. J. Harris, S. T. Bramwell, D. F. McMorrow, T. Zeiske, and K.W. Godfrey, Phys. Rev. Lett. 79, 2554 (1997).

[3] A. P. Ramirez, A. Hayashi, R. J. Cava, R. Siddharthan, and B. S. Shastry, Nature (London) 399, 333 (1999).

[4] S. T. Bramwell and M. J. P. Gingras, Science 294, 1495 (2001).

[5] H. T. Diep, Frustrated Spin Systems (World Scientific , Singapore, 2004).

[6] X. Ke, R. S. Freitas, B. G. Ueland, G. C. Lau, M. L. Dahlberg, R. J. Cava, R. Moessner, and P. Schiffer, Phys. Rev. Lett. 99, 137203 (2007).

[7] T. Lin, X. Ke, M. Thesberg, P. Schiffer, R. G. Melko, and M. J. P. Gingras, Phys. Rev. B 90, 214433 (2014).

[8] S. Scharffe, O. Breunig, V. Cho, P. Laschitzky, M. Valldor, J. F. Welter, and T. Lorenz, Phys. Rev. B 92, 180405(R) (2015).

[9] A. Peretyatko, K. Nefedev, and Y. Okabe, Phys. Rev. B 95, 144410 (2017).

[10] F. Wang and D. P. Landau, Phys. Rev. Lett. 86, 2050 (2001); Phys. Rev. E 64, 056101 (2001).

[11] R. E. Belardinelli and V. D. Pereyra, Phys. Rev. E 75, 046701 (2007); R. E. Belardinelli, S. Manzi, and V. D. Pereyra, Phys. Rev. E 78, 067701 (2008).

[12] Y. Komura and Y. Okabe, Phys. Rev. E 85, 010102(R) (2012)
[13] T. Vogel, Y. W. Li, T. Wüst, and D. P. Landau, Phys. Rev. Lett. 110, 210603 (2013).

[14] M. V. Ferreyra, G. Giordano, R. A. Borzi, J. J. Betouras, and S. A. Grigera, Eur. Phys. J. B 89, 51 (2016).

[15] S.V. Isakov, R. Moessner, and S. L. Sondhi Phys. Rev. Lett. 95, 217201 (2005).

[16] A. Aharony, A. B. Harris, and S. Wiseman, Phys. Rev. Lett. 81, 252 (1998).

[17] M. I. Marques and J. A. Gonzalo, Phys. Rev. E 60, 2394 (1999).

[18] H. Shinaoka, Y. Tomita, and Y. Motome, Phys. Rev. B 90, 165119 (2014).

[19] J. F. Nagle, J. Math. Phys. 7, 1484 (1966).

[20] G. H. Wannier, Phys. Rev. 79, 357 (1950); Phys. Rev. B 7, 5017 (1973).

[21] X. Yao, Solid State Commun. 150, 160 (2010).

[22] K. Matsuhira, Z. Hiroi, T. Tayama, S. Takagi, and T. Sakakibara, J. Phys. Cond. Mat. 14, L559 (2002).

[23] K. Kano and S. Naya, Prog. Theor. Phys. 10, 158 (1950).

[24] C. L. Henley, Can. J. Phys. 79, 1307 (2001).

[25] M. F. Sykes and J. W. Essam, J. Math. Phys. 5, 1117 (1964)

[26] A. Sen and R. Moessner, Phys. Rev. Lett. 114, 247207 (2015). 